\title{
FACTORES INSTITUCIONALES QUE AFECTAN EL DESARROLLO DE LA INDUSTRIA TURÍSTICA EN MAZATLÁN
}

\section{INSTITUTIONAL FACTORS THAT AFFECT THE DEVELOPMENT OF THE TOURIST INDUSTRY IN MAZATLAN}

\section{Jorge Rubén Ibarra-Martínez}

Jefe del Departamento de Investigación y Posgrado, Universidad de Occidente, Carretera a Culiacancito Km. 1.5, Culiacán; Sinaloa.

\section{RESUMEN}

El presente trabajo es una reflexión sobre como las prácticas sociales negativas presentes en las formas de convivencias de los agentes económicos que integran la industria turística de Mazatlán, son el factor determinante para explicar el estancamiento que está experimentando la ciudad en los últimos años. El análisis de este ensayo es realizado bajo un enfoque de la geografía económica, el cual toma en cuenta a las especificaciones y cualidades de un lugar para explicar sus condiciones y potencialidades de Desarrollo.

Palabras clave: Prácticas sociales, Estancamiento económico, Turismo, Institucionalismo.

\section{SUMMARY}

The present work is a reflection of how the negative social practices that are presents in the coexistence forms of the economic agents, who integrate the tourist industry of Mazatlan, are the determining factor to explain the stagnation that is undergoing the city in the last years. The analysis of this essay was realized under the economic geography approach, which takes into account the specifications of a place to explain its conditions and potentialities for Development.

Recibido: 04 de diciembre de 2011. Aceptado: 02 de marzo de 2012. Publicado como ARTÍCULO CIENTÍFICO en Ra Ximhai 8(3): $255-265$
Keywords: Social practices, Economic Stagnation, Tursism, Institutionalism.

\section{INTRODUCCIÓN}

El puerto de Mazatlán atraviesa por una etapa complicada que ha comenzado a quebrantar la actividad económica de mayor arrastre en la ciudad, el turismo. De acuerdo con información proporcionada por la Cámara Nacional de Comercio, Servicios y Turismo de la localidad, tan sólo en el 2011 fueron cerrados 32 establecimientos comerciales ubicados en la principal zona hotelera y turística mazatleca, con lo cual se calcula se afectó a alrededor de unas 300 familias cuyos ingresos dependían directamente de los empleos que se perdieron por el cierre de estos negocios, (Noroestes, 2011).

Los últimos 3 años han sido críticos para el turismo en Mazatlán. Durante este periodo el puerto ha sido perjudicado con la caída en el número de vuelos que llegan a esta ciudad del pacífico mexicano, ya que en el 2010 hubo una disminución de 3 mil 450 vuelos con respecto a la cantidad que arribaron en el 2007. Así mismo, esta tendencia negativa puede observarse en la disminución del número de pasajeros, que para el 2007 fue de personas, pero que para el 2010 la cantidad descendió, (Secretaría de Turismo, 2011). 
Un punto que pone en evidencia el actual estancamiento turístico de Mazatlán, es la baja conectividad aérea que la ciudad mantiene en comparación con otros destinos turísticos de la región, como lo son Los Cabos o Puerto Vallarta, quienes en los últimos 5 años acumularon un tráfico aéreo superior a los 70 mil vuelos, mientras que Mazatlán apenas alcanzó un número promedio de 36 mil vuelos en el mismo periodo de tiempo, esto a pesar, incluso, de que el puerto mazatleco es una ciudad que poblacionalmente es dos veces más grande que Puerto Vallarta y casi 10 veces más grande que Los Cabos, (INEGI, 2010).

Los cruceros son otra muestra de las dificultades que ha tenido que enfrentar la ciudad de Mazatlán para retener la afluencia de turistas que llegan al puerto a vacacionar y disfrutar de sus amenidades. Tan solo entre el 2010 y el 2011 dejaron de llegar 61 cruceros turísticos que tenían programado arribar a Mazatlán, pero que al final cancelaron su visita. Este detrimento ha sido una situación constante desde el 2007, año en el que al puerto llegaron un total de 220 cruceros, para luego descender a 204 en 2008, 164 en 2009 y 150 en 2010, (Noroeste, 2011).

El estancamiento de las actividades turísticas también puede verse reflejado en el bajo porcentaje de alojamiento y en la leve construcción de nuevas habitaciones para colocar a la afluencia turística. Sobre el primer escenario, los números oficiales proporcionados por la Secretaría de Turismo indican que el hospedaje. En lo que se refiere a la construcción de habitaciones, la misma fuente de información señala.

Los datos que aquí se muestran hacen constatar que la ciudad de Mazatlán se encuentra sumergida en un proceso de estancamiento de la que se considera su principal actividad económica y de la cual dependen directamente cerca de 44 mil trabajadores que laboran en el sector hotelero, comercial, restaurante, de transporte y de servicios culturales y de esparcimiento, y que representan el 29.6 por ciento del total de la población económicamente activa de toda la ciudad, (INEGI, 2011).

La finalidad de este trabajo es ofrecer una reflexión sobre las posibles causas del estancamiento de la economía turística en Mazatlán y que impactan en el desarrollo de este puerto y en la calidad de vida de sus habitantes, puesto que, de acuerdo con estimaciones realizadas por Nava e Ibarra, (2009), está actividad representa el 46.89 por ciento del producto Interno Bruto del municipio, es decir, casi la mitad de la riqueza de sus pobladores.

Para este análisis es necesario que primero tengamos claro que las actividades relacionadas con turismo no se desarrollan de manera aislada e independiente, sino que todas ellas forman parte de un esquema productivo de tipo industrial, en el que, como veremos más adelante, las instituciones y convenciones sociales de los actores que ahí participan, son trascendentales para el progreso del sistema turístico en su conjunto.

En este sentido, en este trabajo comenzamos por analizar las repercusiones positivas que tiene una región cuya economía está basada alrededor de un tipo de organización industrial aglomerada. Posteriormente veremos como el turismo puede también ser concebido como una actividad industrial; esto sentará las bases para apreciar como se ha venido construyendo la industria turística en Mazatlán, de la cual, finalmente, analizaremos, los factores más determinantes para su desarrollo. 
La Industria como motor del crecimiento económico regional

Dentro de la historia geográfica del capitalismo es posible constatar que los lugares que en determinado momento han presentado un mayor índice de desarrollo económico, son aquellos que cuentan con una economía basada en las actividades industriales aglomeradas. Definida por Michael Porter (2005), la aglomeración o Cluster, es "un sistema al que pertenecen empresas y ramas industriales que establecen vínculos de interdependencia funcional para el desarrollo de sus procesos productivos y para la obtención de determinados productos". Bajo esta conceptualización, las aglomeraciones son un tipo de organización productiva que se caracteriza por las interrelaciones verticales, horizontales $\mathrm{y}$ colaterales que las empresas aglomeradas producen alrededor de los mercados, las tecnologías y los capitales que las envuelven.

Sin entrar en detalles sobre las crecientes caracterizaciones y tipos de Clustes o alglomeraciones industriales que los teóricos han desarrollado en los últimos años, se puede decir que la importancia de una economía industrial está en función de los altísimos niveles de especialización y competitividad inherentes a estas formas de organización productiva, las cuales llegan a producir altas tasas de crecimiento sostenido, lo que además las lleva a convertirse en el completo motor de arrastre económico de una región, lo cual viene a ser lo que Francois Perroux (1972) llamó Polo de crecimiento.

El crecimiento y desarrollo de una región impulsado por una industria aglomerada, puede explicarse, de acuerdo con Allen Scott (1998) y Michael Storper (1997), debido a los efectos de la división del trabajo que se traduce una variedad de agentes económicos especializados e interrelacionados, los cuales participan conjuntamente en la actividad industrial para que la elaboración del producto sea más eficiente. Es decir, que los agentes de producción de una economía de este tipo se encuentran interconectados en el sentido de que la eficiencia de cada uno repercute en el crecimiento total de la industria y a su vez, las ganancias de cada uno de estos agentes es producto de las ganancias de la industria en su totalidad.

De esta forma es como se explica que con cada ronda de producción, la división del trabajo no solo se hace más compleja y especializada la economía de una región, sino lo más importante es que logra emerger un aspecto cualitativo en el sistema económico de la región a causa del conocimiento, las habilidades y las nuevas técnicas adquiridas en este proceso, lo cual termina por hacer más competitivos a los productos de la región, (Storper y Walker, 1989). Finalmente con la consecuente alza en la demanda externa de los productos de este tipo de regiones convertidas en centros altamente competitivos e innovadores, se termina por producir, de acuerdo con Kaldor (1981), un efecto multiplicador en la economía interna, pues al aumentar la producción aumentan el ingreso y también el consumo y la demanda por servicios.

\section{El turismo visto como Industria}

Al turismo es difícil imaginarlo como una Industria. Cuando se le menciona, comúnmente se le coloca dentro de las actividades del sector servicios. Esta idea está fuertemente influenciada por una concepción tradicional de la economía, en donde se clasifica rígidamente a las actividades económicas y se les cataloga bajo tres categorías inamovibles: la extracción, la transformación y los servicios. Sin embargo, es necesario recordar, que en 
este trabajo, cuando nos referimos a una industria, no lo hacemos en el sentido de fábrica o manufactura, sino que lo hacemos con la intención de detallar al conjunto de actividades económicas organizadas de forma industrial, es decir, a una aglomeración de agentes económicos que producen de manera interdependiente, para un mercado común. Esta postura nos lleva a entender la aparición de industrias postfordistas, flexibles (Powell y Snelmman, 2004), que elaboran productos no tangibles (Madel, 2008), como la industria del Cine, la industria de la música, la industria de la salud, la industria de la educación o la industria del financiamiento, las cuales han llegado a ser el paradigma de las sociedades del conocimiento, (Brinkley, 2006).

Bajo está lógica de entendimiento, Nava e Ibarra (2010) concuerdan en que el turismo puede ser comprendido como "una industria anclada geográficamente cuyo principal recurso es el territorio, entendido este como un espacio de relaciones e interdependencias". Del mismo modo, Ibáñez (2006) menciona que al sector turístico es posible observarlo como al "conjunto de actividades económicas organizadas y encaminadas a satisfacer, por medio de productos y servicios turísticos, la demanda de personas que viajan por placer, negocios u otros propósitos de esparcimiento, hacia un determinado lugar por un perentorio de tiempo".

En una caracterización de la industria turística, la organización de sus actividades puede dividirse de acuerdo a los productos turísticos que cada región ofrece. En este sentido, existen algunos lugares especializados en el turismo cultural, otros en un turismo ecológico, unos más en turismo deportivo, de negocios, de playa y otros más que están relacionados con la oferta que cada punto desarrolla y promueve como atracción para los visitantes.

Así mismo, puede haber una caracterización en base a los servicios que se proporcionan, como el alojamiento, la alimentación, información, entretenimiento y demás. Luego también es factible diferenciar a las industrias turísticas por el tipo de empresas que actúan como agentes económicos, como hoteles, restaurantes, agentes de viaje, o transportistas, los cuales, en conjunto, están encargados de proporcionar el producto turístico a los consumidores del mismo.

Finalmente, para entender a la industria turística como tal, y así diferenciarla de la tradicional concepción que la cataloga como una actividad del sector servicios, es fundamental que se ponga total atención a la relación que se crea entre los agentes económicos que interactúan. Estas relaciones $\mathrm{y}$ formas de convivencia económica, a las que el paso del tiempo las transforma en convenciones e instituciones sociales, son las que caracterizan al lugar, y lo determinan para su desarrollo.

Más tarde en este trabajo nos enfocaremos a analizar el impacto de este tipo de convenciones e instituciones sociales sobre la industria turística mazatleca, no obstante, primero nos abocaremos a describir las características de Mazatlán como industria turística, y para esto nos apoyaremos en dos obras del historiador y sociólogo Arturo Santamaría, la primera titulada El Nacimiento del Turismo en Mazatlán (2002), donde se relatan los albores de esta actividad en el puerto; y la segunda, Del Alba al Anochecer(2005), en la que se documenta la consolidación de la ciudad como destino de sol y playa.

La industria turística en Mazatlán 
La ciudad de Mazatlán emergió debido a una vocación comercial que la llevó prosperar a lo largo del siglo XIX. Durante esta época la principal actividad fue el tráfico de mercancías que se administraban por medio de la aduana, sin embargo, al ser un puerto situado entre una combinación de altos acantilados rocosos y suaves playas de arena clara, el lugar se convirtió sin más esfuerzos que su existencia, en un atractivo para los comerciantes que visitaban la ciudad por motivos meramente económicos, y que a su regreso llevaban consigo historias de un pequeño lugar situado en las costas del mar pacífico.

En el año de 1899 la sociedad mazatleca se organiza por primera vez para realizar las fiestas del carnaval. Este fue el primer paso importante para atraer a más personas que visitaran el puerto con el puro propósito de divertirse. La fiesta del Carnaval atraía principalmente visitantes regionales de Guamuchil, Culiacán, Tepic y otros poblados, luego la invitación se extendió hacía grupos de la ciudad de México y Guadalajara, aprovechando el enlace vía ferrocarril. A lo largo de esos años, las caminatas por las playas y los paseos por calles y plazuelas se convirtieron en los principales atractivos para los visitantes, y aunque el impacto económico era todavía limitado, fue en el sector de Olas Altas donde se construyó en 1923 el primer hotel frente al mar.

Unhechoque es consideradocomoparteaguas importante para el turismo en México, es la creación en 1929 de la Comisión Mixta Pro Turismo, no obstante Mazatlán no se vinculó de inmediato a esta política nacional, y así la vocación turística del puerto se fue dando de manera más paulatina y espontanea, sin un plan estratégico y sin medidas definidas. El turismo se impulsaba por inercia y de acuerdo a las demandas del día a día de los visitantes.

Tras esas primeras experiencias puede establecerse que el turismo como industria en Mazatlán inició hasta la mitad del siglo XX, en una coyuntura donde al fin de la segunda guerra mundial México comenzó a presentar un crecimiento económico importante, y en donde al turismo se le empezó a considerar como una actividad prioritaria para el Desarrollo Nacional. En este escenario, el Presidente Miguel Alemán le otorgó gran importancia a está actividad por advertir en ella un gran potencial económico. Y es precisamente en los años Cincuenta cuando Mazatlán logra los primeros apoyos oficiales por parte del Gobierno Federal, además que pudo beneficiarse de las diversas políticas implementadas a nivel nacional con el fin de promocionar los destinos turísticos del País, como lo fueron el establecimiento de vacaciones escalonadas, o la construcción de la carretera pavimentada desde nogales. De igual forma, a nivel estatal se comenzaron a implantar normas con el propósito de regular esta actividad económica. Por ejemplo en estos años se determino hacer obligatorio en los hoteles los servicios de corriente eléctrica, refrigeración, ascensores y teléfonos.

El impulso inicial que le dieron las políticas de fomento turístico a Mazatlán finalmente logró estimular la construcción de grandes hoteles en la ciudad. Uno de los más emblemáticos fue el Hotel Freeman, que con una arquitectura revolucionaria para ese tiempo y con diseño funcionalista, abrió sus puertas en 1955 como el rascacielos más grande del noroeste de México. También en 1957 el Hotel De Cima inauguraba una nueva zona hotelera sobre la franja costera, donde después aparecerían poco a poco otros hoteles como el Dorado, el Aqua Marina, el Arenas, el Olas Altas y posteriormente el Hotel Hacienda en 1967. De igual forma 
en este periodo se abrieron nuevos bares y restaurantes como La Copa de Leche, El Joncols, El Barco, El Palomar, El Shrimp Bucket y El Señor Frogs.

El periodo de consolidación para el turismo en Mazatlán ocurrió en la década de los setentas. Estos años se destacaron por ser la mejor época turística del puerto, llegándose a posicionar en 1973 como el segundo destino de Sol y Playa más importante del País. Aquí el afianzamiento del turismo estuvo guiado por la expansión del Paseo Costero, la inauguración de la avenida Camarón Sábalo, y sobre todo por la construcción de la Zona Dorada, una nueva franja totalmente turística donde se comenzaron a erigir hoteles como Islas del Sol, Las Palmas, El Camino Real y el emblemático Hotel el Cid. Al finalizar este auge de la construcción que tuvo Mazatlán entre 1970 y 1976, se lograron contabilizar 137 hoteles además de 17 campos para remolques, 89 restaurantes y 34 bares o restaurantes. Además, debido a la incesante demanda turística, esta etapa no solo se caracterizó por la construcción de nuevos hoteles, comercios y restaurantes se aceleró, sino que también se incrementó el número de aerolíneas internacionales como Bonanza Airlines, Southwest o Wester Airlines, que conectaron de forma directa al puerto con algunas ciudades de Estados Unidos.

A principios de los ochentas México se vio envuelto en un contexto de crisis económica de la que Mazatlán logró esquivar debido a que el turismo se había consolidado como una industria de arreste económico. En estos años el destino consiguió permanecer como el segundo principal puerto turístico del país, esto se debió en parte al núcleo de fieles turistas que lo visitaban año tras año, pero también porque los empresarios mazatlecos mantuvieron tarifas económica a pesar de la difícil situación que imperaba en el país, esto como una estrategia para convertir al puerto en el centro turístico con los precios mas bajos de todo México. Sin embargo esto sólo funciono a corto plazo porque a la larga Mazatlán perdió competitividad ante los nuevos centros turísticos como Los Cabos, Cancún y puerto Vallarta que durante las próximas décadas atraerían a visitantes de un poder adquisitivo mayor.

Otro de los aspectos importantes de la actividad turística de Mazatlán en los ochentas, es que la promoción, contrario a los años anteriores donde el gobierno jugaba el papel central, ahora comenzó a recaer en el sector empresarial constituido por los agentes de viajes, los hoteleros y las líneas aéreas. Estos empezaron a implantar nuevas promociones turísticas como los paquetes que incluyen transporte aéreo, hospedaje y alimentos, también se intensificaron las ventas de tiempos compartidos, incursionaron los primeros vuelos charters $\mathrm{y}$ arribaron los primero collage tours en las vacaciones de primavera.

Con la incursión de otros destinos turísticos a nivel nacional, Mazatlán fue perdiendo competitividad, lo que urgió a realizar esfuerzos para diferencia el producto turístico que se ofrecía. Es por eso que a finales de los años ochentas se inició el Programa Para el Rescate, Revitalización y Conservación del Patrimonio del Centro Histórico de Mazatlán, con lo cual se buscó establecer actividades comerciales, culturales en esta zona que para estos años ya estaba muy deteriorada. Con estas obras dentro de las cuales sobresalía la remodelación del teatro Ángela Peralta, se consiguió presentar por primera vez una alternativa aparte de los bares, restaurantes y centros nocturnos.

La década de los noventa auguraba buenos 
tiempos para la economía de Mazatlán, sin embargo, a pesar de las buenas expectativas por parte de los empresarios, esta nueva década marcó el declive turístico en el puerto, que junto con el descalabro estrepitoso del conjunto de los demás principales renglones económicos de la ciudad, (portuario y el pesquero), terminaron por ocasionar para finales de 1993, el cierre de 400 comercios y empresas. Para el año siguiente, durante Enero y Febrero, que son los meses que tradicionalmente han sido considerados de temporada alta en el turismo extranjero, la ocupación hotelera fue de tan solo $30 \%$ y los vuelos provenientes del extranjero operaban con números muy bajos. Por si fuera poco se derrumbaron proyectos tan esperados como el de la marina, al quebrar la compañía constructora perteneciente al Grupo SIDEC, que se vio afectado por la crisis de 1994.

Con la entrada del nuevo siglo las actividades económicas portuaria y pesquera en Mazatlán han seguido en declive. Sin embargo, durante últimos estos años, si bien no se han caracterizado por un repunte impresionante en el turismo, este si se ha posicionado como la actividad que mas arrastre económico tiene para la ciudad de Mazatlán. Recientemente Mazatlán ha tratado de reposicionarse con nuevos proyectos como el de Estrella de Mar en la Isla de la piedra o el Plan Nuevo Mazatlán y las recientes inversiones inmobiliarias en el área de la marina le han dado nuevos aires a la industria turística.

Actualmente Mazatlán ha diversificado sus producto turísticos, ahora además de la playa y el carnaval, cuenta con turismo deportivo con el maratón internacional pacifico, el triatlón, torneos de surf y pesca deportiva. También ha reforzado el turismo cultural el cual gira alrededor del centro histórico, la Plazuela Machado y el Teatro
Ángela Peralta. Además el área geográfica de la industria turística mazatleca se ha extendido mas allá del puerto, ahora cuenta con un alcance regional que ha diversificado aún más sus productos turísticos en todo el sur de Sinaloa, con los recorridos a pueblos cercanos como San Ignacio, El Quelite, Concordia, Copala, Villa Unión, El Roble Rosario, Chametla y las playas de Escuinapa y Teacapán.

Con la crisis en el sector pesquero en los últimos años y el fracaso en hacer de Mazatlán un puerto de embarque comercial de altura, la industria turística se ha colocado como el motor principal de la economía mazatleca, sin embargo la estancamiento de está actividad en los últimos años hace un llamado a entender las causas que han ocasionado que Mazatlán no despunte como destino turístico, especialmente si se compara con otros destinos turísticos como Cancún, Puerto Vallarta, Los Cabos o Acapulco.

Algunas explicaciones al estancamiento de la industria turística en Mazatlán

Dentro de las explicaciones del porqué del estancamiento de la industria turística en Mazatlán, políticos y empresarios comúnmente hacen referencia a factores económicos relacionados con la ubicación, el capital, los precios de la competencia, la infraestructura. El empresario hotelero Julio Berdegué explicaba por ejemplo, que la mayor competitividad de otros destinos se debía a que estos estaban haciendo promociones por debajo de los costos. Estas interpretaciones han llevado a establecer medidas con soluciones a corto plazo como lo han sido la reducción de los precios en las tarifas aéreas y de hospedaje, en 1994 por ejemplo, se llegaron a ofrecer vuelos redondos desde Los Ángeles a tan solo 162 dólares. 
La implementación de estrategias dirigidas a abaratar costos para hacer más competitiva una región está fuertemente influenciada por las teoría clásicas de la economía geográfica clásica, Von Thunen (1826), Weber (1909), Losch (1941), Christaller (1933), y Plander (1935), en donde el desarrollo de una región depende básicamente de dos variables: los recursos y la ubicación. Esto quiere decir que una región mientras mas recursos tenga y conforme tracen una distancia óptima con respecto al mercado u otros recursos, tendrá las mejores condiciones para desarrollar la actividad económica, que de acuerdo con sus ventajas comparativas y competitivas le convenga.

Bajo está lógica, mucho se ha argumentado que el retraso en la culminación de la carretera que conectará a Mazatlán con el este mexicano y el suroeste norteamericano, es una traba que está impidiendo el despegue económico y turístico de la ciudad. Sin embargo, esto no explica como destino como Vallarta, Cancún o Los Cabos, cuya ubicación geográfica es un poco más aislada que la de Mazatlán, cuentan con mayor afluencia turística y de inversión que la del puerto del pacífico.

Por otro lado, con respecto a los recursos, las teorías clásicas también consideran que estos se pueden dividir en: recursos naturales, capital y capital fijo, y que mientras más abundancia de recursos exista en una región, mayores oportunidades de desarrollo se presentarán. Sin embargo, en el ámbito de los recursos naturales, Mazatlán cuenta con un ecosistema de playa de aguas tranquilas y con una temperatura tropical muy agradable incluso en los meses invernales.

De igual forma los recursos de capital nunca han estado ausentes en el ámbito turístico, siempre han existido extranjeros o empresarios locales dispuestos a invertir en la promoción de este puerto de playa o en la construcción de la infraestructura, lo cual se ve reflejado en una gran variedad de hoteles, bares, restaurantes y centros nocturnos.

El capital fijo aquí, también es otro incentivo para su desarrollo., Mazatlán cuenta no solo con un malecón de 21 kilómetros frente al mar, campos de golf, un embarcadero para barcos de pesca deportiva, una marina para yates de lujo, una infraestructura portuaria que recibe cruceros internacionales, carreteras y un aeropuerto que lo conectan con cualquier parte del mundo. Entonces, como explicar el estancamiento a pesar de presentar una riqueza en recursos.

Lo que se intenta demostrar aquí, es que los factores económicos como el de los recursos o la ubicación no pueden ser tomados como única referencia al dar una explicación sobre la situación de la industria turística en Mazatlán. Pues bajo este esquema nunca pudiéramos encontrar respuesta satisfactoria al porqué los turistas dejan de visitar a Mazatlán, o porqué las aerolíneas retiran sus vuelos. No intento dejar fuera a los factores económicos, pero sí queremos dar una respuesta completa a estas incógnitas, se debe poner atención a los factores endógenos, a las circunstancias internas y a su relación con el turismo.

Factores institucionales que afectan la Industria turística en Mazatlán

El espacio económico de una industria no puede ser entendido solo como un punto cartográfico donde se localizan los actores económicos que se relacionan únicamente en base a factores de costos. El espacio económico es mas complejo, pues quienes lo integran, aunque sean agentes económicos y actúan de acuerdo a la maximización de ganancias, tambien son seres sociales con una 
identidad, costumbres, ideales y actitudes que finalmente vienen a impactar negativa o positivamente en el desempeño del sistema económico en conjunto, (Perroux, 1972).

De acuerdo con las teorías institucionalistas, los aspectos no económicos son un factor clave en el desarrollo de una región y su productividad económica, pues esta serie de actitudes y valores son las que arropan al sistema económico.

Sobre este aspecto Canobbio e Ibarra (2007) comentan que "El desarrollo económico de una región, depende de una combinación de elementos técnicos, institucionales y culturales... algunos de ellos, anclados en la tradición y otros en estructuras modernas".

Dentro de los marcos institucionales los teóricos Stiglitz (1994), (Storper, 1997), Putman (2000), Asheim (2002) afirman que pueden existir ciertas convenciones sociales entre los agentes económicos de una industria, que favorecen el desarrollo económico de una región. En este sentido, este enfoque resalta los beneficios de la actuación de los pequeños grupos de afinidad para conformar coaliciones económicas y generar vienes públicos, organizar la participación en los mercados, consolidar redes con altos grados de confianza que reducen el costo de transacciones, limitan las desigualdades y favorecen la formación de grupos de interés.

Por otro lado, otras teorías institucionalistas Snack y Keefer, (1997), Guiso (2004), Durlaf y Fafchamps (2004), también señalan que en una región económica también puede haber formas de convivencia negativas como los caciquismos, clientelismos, nepotismos, corrupción, competencias desleales, o aptitudes adversas al respeto de la propiedad, la democracia, la rendición de cuentas, el estado de derecho, el cuidado del ambiente, que desaniman la inversión, que entorpecen el emprendedurismo, generan desconfianza, elevan los costos de transacción y crean un clima de incertidumbre para el desarrollo.

Es en esta última categoría en la que nos queremos enfocar para explicar lo que pudiera estar ocurriendo en Mazatlán, ya que a lo largo de una vida de casi 30 años en la ciudad, hemos atestiguado como se han formado ciertas convenciones sociales entre quienes participan en la industria turística de la ciudad, y que vienen a entorpecer su desarrollo. A continuación revisamos algunas de las que nos parecen fundamentales.

\section{Divisionismo}

Uno de los grandes problemas del sector empresarial en Mazatlán es la falta de unidad para emprender y apoyar proyectos de beneficio para toda la sociedad. Esta característica ha sido una de las principales causantes del atraso económico en este litoral. Quizá una de las divisiones más conocidas es la de los hoteles, quienes en los años 90 atravesaron una de las crisis más severas por cuestiones de adeudos de cuotas. El conflicto empezó porque un grupo de hoteleros de la asociación de Hoteles y Moteles de Sinaloa reclamaba el acaparamiento del 80 por ciento de los recursos para las campañas publicitarias por parte de un par de hoteles de esta asociación. Al finalizar el grupo que reclamaba los abusos, termino conformando su propia asociación: la "Asociación Tres Islas". Esto ha traído como consecuencia la difícil aplicación y recolección de los impuestos por lo complicado que resulta poner de acuerdo a ambos bandos.

Desafortunadamente esta dicotomía se ha venido profundizando: los habitantes de la "Zona Dorada" y los de las marismas; taxistas 
rojos, ecotaxis verdes, "aurijas","pulmonías" y "Zafaris", que se enfrascan en una disputa permanente por levantar el pasaje; cuatro colegios de abogados, como de ingenieros y peritos valuadores, lo cual marca el fuerte divisionismo entre los gremios de profesionistas. Son rasgos muy acusados todos éstos de atomización social, que se traducen en una perversión de las relaciones ciudadanas que degradan la convivencia de la sociedad mazatleca.

\section{Conflictos violentos}

En Mazatlán los conflictos son constantes entre los guías turísticos, los vendedores de tiempos compartidos. Pero es el enfrentamiento entre los transportista el que mas daño le ha causado al turismo y el que ha evidenciado la impunidad y la falta de cultura cívica mazatleca. Este pleito data desde 1975 pocos años después de haberse construido el aeropuerto Rafael Buelna, cuando se enfrentaron los taxistas de ATAMSA, que laboran en el aeropuerto contra aquellos que trabajan en la ciudad. El problema se suscitó porque los taxistas de ATAMSA tienen placas federales, y de acuerdo con una norma estatal estos no debían recoger pasaje dentro de la ciudad. Entonces cada vez que lo hacían, los taxistas locales bloqueaban las unidades de ATAMSA para impedir que llevaran a los turistas al aeropuerto. En el año 2004 el primer Tribunal Colegiado de Circuito concedió el derecho de ATAMSA para recoger turistas dentro de la ciudad, pero los taxistas locales, sin reconocer la sentencia emanada por el tribunal, continúan con los bloqueos. En los últimos años los bloqueos por parte de los taxistas locales se han extendido también a los autobuses charteros y según el periódico el Debate llegan a ser de 5 por semana en las temporadas altas. Los bloqueos no solo han ocasionado mala imagen para Mazatlán, sino que también han tenido un impacto negativo, pues ya ha habido retiro de vuelos charters, se han retirado empresas promotoras canadienses y además a habido amenazas de retiro por parte de Alaska Airlines, así como de algunos publicistas de no volver a celebrar sus congresos en Mazatlán.

\section{Desorden urbano}

El desorden y la mala planeación también se ha convertido en un hábito al momento de imaginar el entorno urbano de Mazatlán y sus implicaciones hacia el turismo. Esto pudiera parecer normal, pues como en cualquier ciudad de México, la influencia de lo estudios urbanos y la implementación de políticas sobre planeación urbana han llegado tarde. Pero en la ciudad de Mazatlán es inconcebible que por ejemplo, la planta para tratar aguas residuales, la hallan ubicado en la base del cerro del Creston, junto a una de las atracciones turísticas mas promocionada: El Faro, y de las instalaciones de flotas dedicadas a la pesca deportiva; pero además, es el punto de entrada de los turistas de cruceros que arriban al puerto percibiendo los malos olores que despide la planta.

Otro de los ejemplos del desorden urbano es la construcción de una planta de generación eléctrica y de un edificio para el sindicato de maestros dentro de los terrenos del "Bosque de la Ciudad", como también los permisos que otorgó el municipio recientemente, para la colocación de un corredor automovilístico, el levantamiento de unos condominios lujosos y la construcción de una tienda comercial dentro del espacio la laguna del camarón. Además es inexplicable como siendo las playas lo mas atractivo de Mazatlán, existan tuberías que arrojan el drenaje al mar en el área del malecón y la "Zona Dorada". De igual forma, el hábito colectivo de tirar basura en las calles y el 
no solucionar los problemas de drenaje e inundación de calles en plena zona hotelera, demuestran el caos urbano que se padece en Mazatlán y que es tan pernicioso para el desarrollo de la actividad turística.

\section{Violencia y cultura del narcotráfico}

Entre las formas de convivencia mas dañinas que han afectado al turismo en Mazatlán, ha sido la violencia que impera en la región sinaloense por la situación del narcotráfico. La ciudad de Mazatlán siempre ha sido muy permisiva con la llamada cultura del narco, pues es común ver en la temporada alta cómo grupos de delincuentes disfrazados de turistas, se apropian de la zona turística implantando el desorden sin que ninguna autoridad les contenga. Es en Semana Santa, la fecha de mayor afluencia turística para Mazatlán, la época mas propicia para que los narco-juniors de todo el estado se den cita en la avenida del Mar y en la Zona Dorada para alimentar su ego y se hagan acompañar con las estruendosas bandas musicales de la región, luciendo sus costosos autos de lujo, cierren por las noches los antros de moda para ellos solos, e incluso atraviesen sus poderosas unidades en medio del infernal aforo vehicular y revienten prácticamente el tráfico por toda la zona costera, bajo la mirada complaciente de las autoridades y la expectación morbosa de los ciudadanos.

Más allá de una permisividad y empatía por la narco-cultura, Mazatlán ha experimentado en los últimos años un incremento en los índices de violencia, en la que ya es común que turistas sean víctimas de enfrentamientos entre distintos grupos delictivos que operan en la ciudad. La violencia en Mazatlán también ha incrementado en cuanto a formas innovadoras de crear terror, (decapitados, colgados, descuartizados), ante esto la ciudad obtiene cada día más una reputación de criminalidad, tanto así que en los índices internacionales Mazatlán aparece como una de las ciudades más peligrosas de todo el mundo, lo cual por supuesto ha levantado alarma en los turistas e incluso recientemente el Gobierno de los Estados Unidos advertía a sus habitantes que no viajaran al puerto de Mazatlán.

\section{CONCLUSIONES}

La industria turística en Mazatlán se ha posicionado en los últimos años como la actividad que más beneficios económicos le aporta. De acuerdo con factores económicos como la infraestructura y el capital, Mazatlán cuenta con ventajas competitivas en el turismo, además sus atractivos naturales le han hecho posible ofrecer una gran variedad de productos turísticos y expandir su alcance geográfico hasta un nivel regional. Pero a pesar de tener estas ventajas, resulta paradójico que Mazatlán experimente un rezago con respecto a otros destinos turísticos que arrancaron mucho después.

Una de las causas de este rezago, es debido a que se ha visto al espacio económico mazatleco solo como un contendor en el que solo localizar recursos es suficiente para que funcione el sistema económico, un espacio donde es necesario solo poner un aeropuerto, carretera y hoteles para que los turistas llegaran por si solos. Pero para que una industria del turismo pueda convertirse en un verdadero motor de arrastre que promueva el desarrollo en Mazatlán, es necesario estar conscientes que las convenciones, la ideología y las instituciones condicionan el funcionamiento económico. En este sentido era necesario observar las características sociales, institucionales y organizativas que envuelven a la industria turística de Mazatlán. Y entones ahora debemos replantearnos la pregunta de la siguiente forma, ¿Son favorables para su desarrollo?, 
a la conclusión que hemos llegado en este ensayo es que no.

Que Los hábitos conflictivos y de división, la violencia, la forma de organización, las instituciones mediocres, la apatía y los liderazgos perniciosos que se presentan en Mazatlán, han socavado a la industria turística.

\section{LITERATURA CITADA}

Asheim, B.T. 2002. Temporary organization and special embeddedness of learning and knowledge creation, Geografiska Annaler: Series B, Human Geography, Vol. 84.

Brinkley, Ian. 2006. Defining the knowledge Economy, The Work Fundation.

Canobbio, Claudia y Guillermo Ibarra. 2007. Sistemas de innovación regional en clusters biotecnológicos de Canadá, Casa Juan Pablos, México.

Christaller, Walter. 1933. Diez Zentralen orte in suddeutschaland, Gustav Fischer, Jena.

Durlaf y Fafchamps. 2004. Social capital, working paper, Cambridge, Mass. National Bureau of Economic Research, Economy, Vol. 99.

Madel, Michael. 2008. The U.S. Economy's Best Bet: The Intangible Sector, Bussinesweek.

Nava Miriam y Guillermo Ibarra. 2010. Actores locales y competitividad turística de Mazatlán. El territorio como producto turístico, Topofilia, El Colegio de Sonora Vol. II.

Perroux, Francois. 1972. Note on the concepto of "Growth Poles". McKee, David L., Dean.

Porter, M. 1990. The CompetitiveAdvantage of Natios.New York. The Free Press.

Powell Walter y Kaisa Snelmman. 2004. The knowledge economy, Stanford University, Stanford.

Putman, R. 2000. Bowling Alone: The collapse and revival of american community, New York: Simon and Schister regionalism, Regional Development Studies, Vol. 7.

Robert, Leahy William H. (Edrs). Regional Economics: Theory and Practice. (93-102).

New York. The Free Press.

Santamaría Gómez, A. 2005. Del alba al anochecer. El turismo en Mazatlán, 1972-

2004. México. Universidad Autónoma de Sinaloa.

Santamaría Gómez, A. 2002. El nacimiento del turismo en Mazatlán, 1923 -1971.

Culiacán. México. Universidad Autónoma de Sinaloa.

Scott, Allen J.1998. Regions and the world economy. The coming shape of global production, competition, and political order, Oxford University Press.

Storper, Michael y Richard Walker. 1989. The capitalist imperative. Territory, technology, and industrial growth, Basil Blackwell, Oxford, Reino Unido.

Storper, Michael. 1997. The regional world. Territorial development in a global economy, New York, The Ghilford Press.

Thunen, Von. 1966. Isolated state. An introduction by Peter Hall, Pergamon Press Oxford, Nueva York.

Weber, Alfred. 1929. Theory of location of industries, The University of 
Chicago Press, Chicago.

Jorge Rubén Ibarra Martínez, Cuenta con estudios de Doctorado en estudios de América del Norte, y Maestría en Estudios de Estados Unidos y Canadá, por la Universidad Autónoma de Sinaloa. Ha realizado estancias académicas y de investigación en la Universidad de California en Los Ángeles, y en la Universidad de Illinois. Actualmente es miembro del Sistema Sinaloense de Investigadores y Tecnólogos, y labora para la Universidad de Occidente como Jefe del Departamento de Investigación y Posgrado. 